\title{
Simulation of Space Charge Effects in Resistive Plate Chambers
}

\author{
Christian Lippmann and Werner Riegler
}

\begin{abstract}
Multigap resistive plate chambers [1] with $0.3-\mathrm{mm}$ gas gaps operated in avalanche mode at atmospheric pressure have reached timing accuracies below 50 ps (standard deviation) with efficiencies above 99\% [2]. The avalanches in high homogeneous electric fields of $100 \mathrm{kV} / \mathrm{cm}$ are strongly influenced by space charge effects which are the main topic of this paper. We extend a previously discussed Monte Carlo simulation model of avalanches in resistive plate chambers [3] by the dynamic calculation of the electric field in the avalanches. We complete the previously presented results on time resolution and efficiency data with simulated charge spectra. The simulated data shows good agreement with measurements. The detailed simulation of the avalanche saturation due to the space charge fields explains the small observed charges, the shape of the spectra, and the linear increase of average charges with high voltage.
\end{abstract}

Index Terms - Charge spectra, resistive plate chambers (RPCs), simulation, space charge effect.

\section{INTRODUCTION}

$\mathbf{R}$ ESISTIVE plate chambers (RPCs) were originally operated in streamer mode providing large signals which simplifies readout electronics and gap uniformity requirements. However, high rate applications and detector aging issues made the operation in avalanche mode popular. This was also facilitated by the development of new highly quenching $\mathrm{C}_{2} \mathrm{~F}_{4} \mathrm{H}_{2}$-based gas mixtures [4]. While the physics of streamers is difficult to study, the avalanche mode opened the possibility for a detailed simulation of the detector physics processes in RPCs.

In this paper, we discuss the avalanche propagation, charge spectra, and average avalanche charges in timing RPCs, using the devices by Fonte et al. [2], [5], [6] as examples. They have $0.3 \mathrm{~mm}$ gas gaps and resistive glass plates with a volume resistivity of about $2 \times 10^{12} \Omega \mathrm{cm}$. The gas is $\mathrm{C}_{2} \mathrm{~F}_{4} \mathrm{H}_{2} / i-\mathrm{C}_{4} \mathrm{H}_{10} / \mathrm{SF}_{6} 85 / 5 / 10$ and the operating voltage is 6 (3) $\mathrm{kV}$ for the double (single) gap RPCs, resulting in an electric field of $100 \mathrm{kV} / \mathrm{cm}$ in the gas gaps (Fig. 1).

In order to explain the high efficiencies of RPCs, a large ionization density and large gas gain are required. A large ionization density is necessary to ensure a high probability for the deposit of electron clusters close the cathode. From here the avalanches can cross a large fraction of the gap and due to the exponential growth they can reach sufficient sizes to be detected. Even in the case of a large ionization density the gas gain has to be extremely large to ensure that enough avalanches reach

Manuscript received November 7, 2002; revised June 27, 2003.

The authors are with the EP-Division, CERN, CH-1211 Geneva 23, Switzer-

land (e-mail: Christian.Lippmann@cern.ch; Werner.Riegler@cern.ch).

Digital Object Identifier 10.1109/TNS.2003.814536
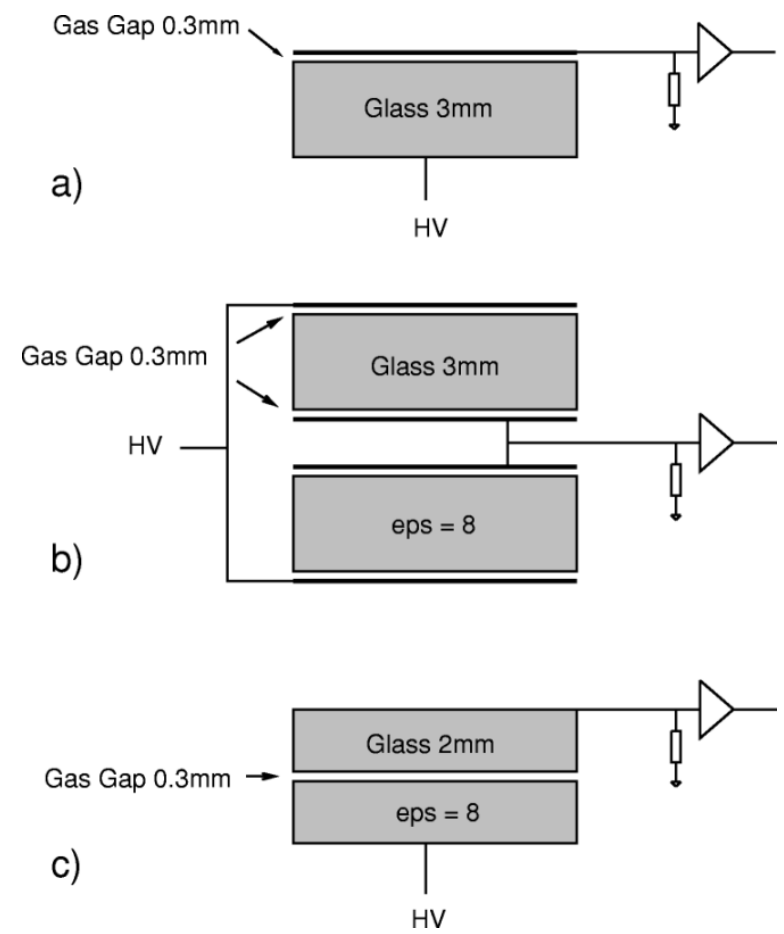

Fig. 1. RPC geometries similar to the ones developed by Fonte et al. [2], [5], [6].

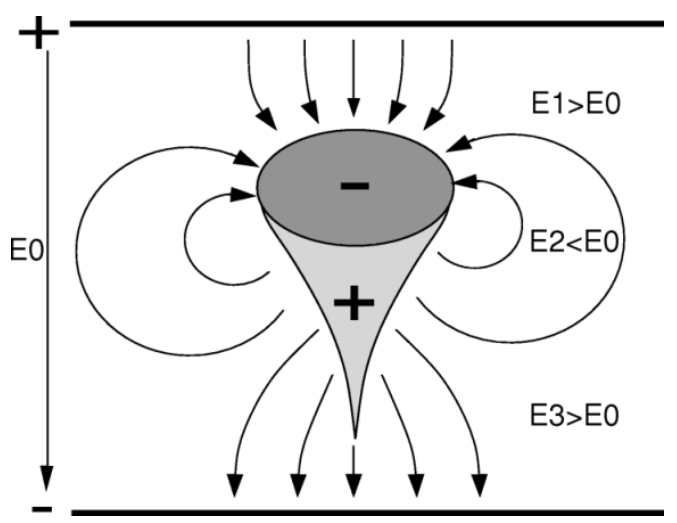

Fig. 2. A schematic image of an avalanche in a parallel plate geometry [7]

the threshold and thus explain the measured RPC efficiencies. In that case, only a strong space charge effect would explain the observed small avalanche charges of around $1 \mathrm{pC}$. The influence of the electric field of the avalanche charges (the influence of the space charge) on the applied electric field $E_{0}$ in the gas gap of an RPC is shown schematically in Fig. 2. The electric field $E_{2}$ sensed by the majority of the moving electrons in the center of the avalanche is reduced. At the tip and tail of the avalanche 
the fields $E_{1}$ and $E_{3}$ are increased. It is not obvious whether an avalanche can progress under the extreme conditions necessary for the suppression of high charges without developing into a streamer at one of the points where the field is increased [7].

Previous simulations show that the measured efficiencies and time resolutions of timing RPCs can be reproduced using a simulation model that assumes a uniform electric field equal to the applied field [3]. However, the simulated avalanche charges differ from measurements by a factor of up to $10^{7}$ and the obtained charge spectra show an exponential shape while measurements show a very different shape. (For example, see [6].) In this paper, we extend the model from [3] by the inclusion of the space charge effect, by dynamically calculating the electric field of the avalanche charges and adding it to the applied electric field [8]. We shall see that a careful study of the electric field deformations in the avalanche can accurately explain the small observed charges.

\section{Simulation OF Timing RPCS}

Monte Carlo simulation procedures of RPCs have been reported in [3] and [8]-[10]. As mentioned previously, our simulation procedure bases on the one described in [3]. The input parameters are

- average distance between primary clusters $\lambda$;

- probability distribution for the number of electrons per cluster;

- Townsend coefficient $\alpha$;

- attachment coefficient $\eta$;

- drift velocity $v$ of electrons in the gas;

- transverse and longitudinal diffusion coefficients $D_{t}$ and $D_{l}$.

These parameters and their dependence on electric field and pressure are obtained by the programs HEED [11], MAGBOLTZ [12], and IMONTE [13]. Details are given in [3]. While the electrons in the gas gap drift toward the anode, their multiplication will statistically fluctuate around an average given by an exponential law: $n(\delta z)=\exp ((\alpha-\eta) \delta z)$. The simulation employs a one-dimensional model in the sense that the propagation of the avalanches is simulated on a line (the $z$-axis) through the gas gap, which is divided into several steps. The new ingredient is the calculation of the $z$-component of the electric field at each step where electrons are found and after each time step [8]. For the field of the space charge, we use analytic formulas for the potential of a point charge in an infinite plane condenser with three homogeneous dielectric layers [14], [15]. The space charge at each step is contained transversely in disks with Gaussian charge distributions. The standard deviation of the Gaussians depends on $D_{t}$ and the drift distance. With the value of the electric field we calculate the values of the parameters $\alpha, \eta$ and $v$ at each step.

The induced current on a pickup electrode is

$$
i(t)=\vec{E}_{w} \cdot \vec{v}(t) q N(t)
$$

where $\vec{E}_{w}$ is the normalized weighting field and $N(t)$ is the number of charge carriers moving with velocity $v(t)$. For analytic formulas for the weighting field in an RPC see [14], [16].
If we accept that a space charge effect limits the maximum charge carried in an avalanche, which can be simulated in a crude way by cutting the avalanche growth at a certain size $\left(\approx 1.6 \times 10^{7}\right.$ electrons for the discussed RPC geometry), we find satisfactory results [3], [17] also for the charge spectra [4]. In this paper, we shall present results using the more correct treatment of saturation by including the actual electric field of the space charge.

\section{AVALANCHES IN RPCS, DiscusSiON}

As discussed previously, the electric field of the positive and negative avalanche charges reduce the field sensed by the electrons in the center of the avalanche. A lowered field strength reduces the effective Townsend coefficient $\alpha_{\text {eff }}=\alpha-\eta$ and therefore the gas gain. $\alpha_{\text {eff }}$ can even reach negative values leading to the attachment of free electrons.

A simulated avalanche consisting of three primary clusters in a single gap timing RPC is shown in Fig. 3. The first two clusters have merged by diffusion at $0.61 \mathrm{~ns}$. Here, we also observe that the space charge begins to alter the electric field in the gap. As in Fig. 2, the field is lowered in the center of the electron cloud and increased at its tip and tail. At $0.91 \mathrm{~ns}$, we observe that the field at the center of the electron cloud still remaining in the gas gap is lowered to half the applied electric field value $E_{0}$, while at the tip, its value is more than doubled. The drift velocity at the tail of the electron cloud is lowered such that the electron distribution is becoming wider. After this stage, the electrons moving in the gap are all in a region of decreased field strength. This is due to the amount of positive ions remaining in the gap (they are drifting much slower than the electrons), but also the electrons that reached the anode stick on the resistive surface and lower the field just before the anode. At this stage, we find strong attachment and small drift velocities. (See image at $1.04 \mathrm{~ns}$ in Fig. 3.) The process of electron attachment creates negative ions. After all electrons either left the gas gap or got attached, we find a majority of negative ions just before the anode. (See image at $1.58 \mathrm{~ns}$ in Fig. 3.)

Simulated spectra are shown in Fig. 4. The saturation effect due to the space charge field suppresses the large charge values and leads to a shape of the spectrum that is different from exponential. The average charge of around $2 \mathrm{pC}$ is close to measured charges and also the shape of the spectrum matches experimental results very well (For example (see [6]).

The assumption that the transverse dispersion of the avalanche is given only by diffusion is certainly underestimating the real radial spread of the electrons, especially at a later stage, where a strong space charge effect will lead to repulsion of electrons in the avalanches head. This is also clear from Fig. 2. A detailed study of the radial space charge fields would clarify the effects on the avalanche propagation but lies beyond the scope of this article.

\section{A. Streamers}

The phenomenon of streamers in RPCs is discussed in detail in [7]. Anode streamers form at the tip of a moving cluster of electrons in an avalanche while cathode streamers form at its tail [18]. 

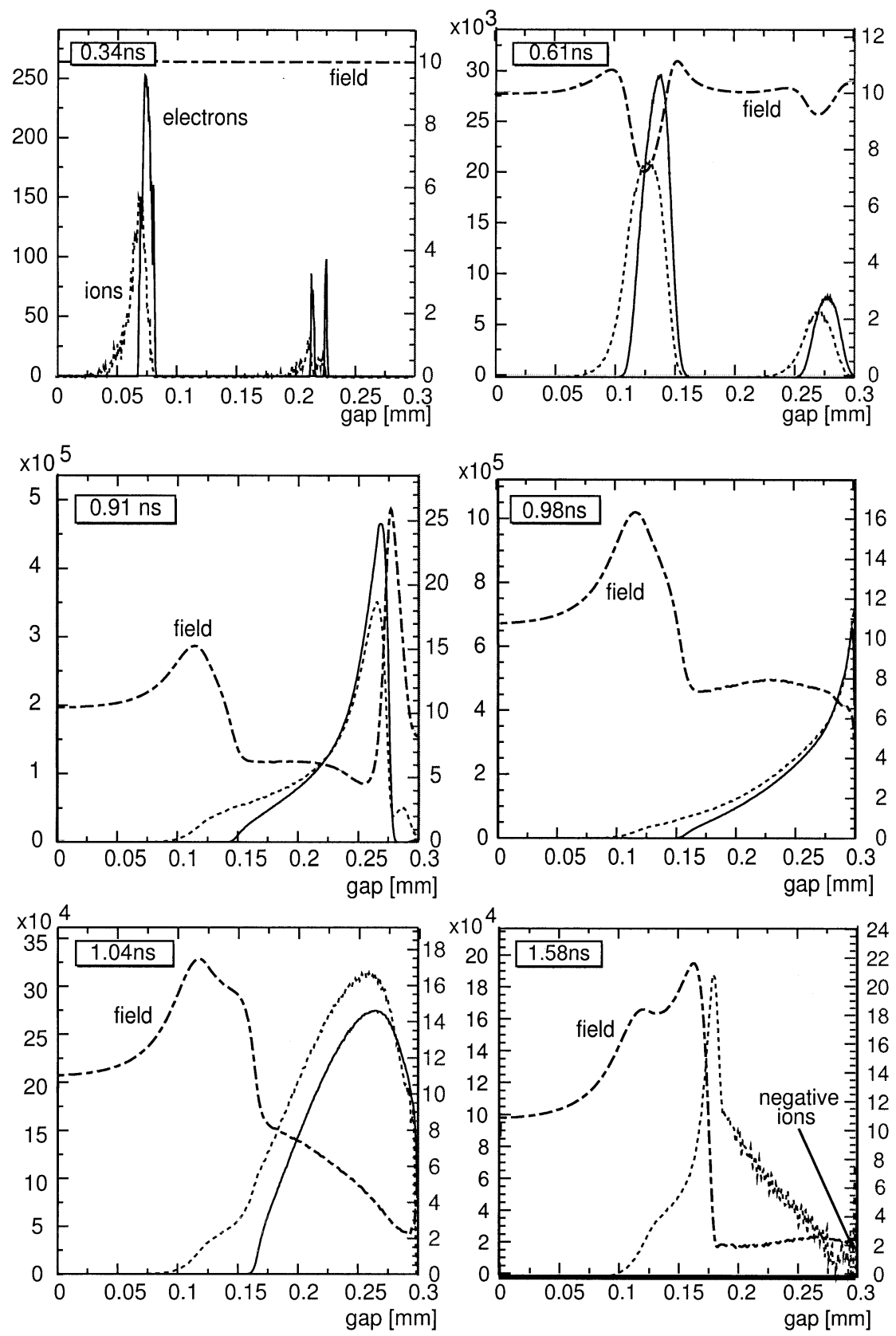

Fig. 3. A simulated avalanche. We show snapshots of the charge configuration in a $0.3 \mathrm{~mm}$ single gap. The distribution of positive ions, negative ions and electrons at different time steps is shown and corresponds to the axes on the left (number of charges per step). The gap is divided in 500 steps. The electric field is also plotted (axes on the right; $\mathrm{kV} / \mathrm{mm}$ ). The applied high voltage is $3 \mathrm{kV}$, pressure $p=1013 \mathrm{mb}$, and temperature $T=296.15 \mathrm{~K}$.

The presence of a big space charge is a necessary requirement for the development of a streamer. This space charge can be either ions from previous avalanches, that have not yet left the gas gap or the charge carriers of the avalanche itself. The second phenomenon leads to the avalanche itself being the cause of its instability. The field of its space charge at some point exceeds some critical value so that the more or less well regulated avalanche propagation transforms into a streamer. When streamers reach both electrodes a channel of high conductivity is formed between the electrodes, leading to a local discharge in the RPC [19].

Fig. 5(b) shows the evolution of the peak value of the electric field strength in the RPC gap in simulated avalanches. We see that the field can easily reach double or three times the value of $E_{0}$. At very high fields, where measurements show a significant streamer probability, the simulations still show a saturated avalanche. Measurements show that at applied field strengths of around $10 \mathrm{kV} / \mathrm{mm}$ in four gap timing RPCs, streamers occur with a probability of around $1 \%$, typically releasing a pulse of $20 \mathrm{pC}$ [2], [5]. At higher voltages the streamer probability is growing up to $25 \%$ at around $11 \mathrm{kV} / \mathrm{mm}$. Therefore, the quantitative description of the avalanche-to-streamer transition is not reproduced by our model. Note that no photonic effects have been included.

\section{B. Space Charge Mode and Streamers}

From wire chambers filled with a quench gas with good UV absorption it is known that for certain high voltages one ob- 


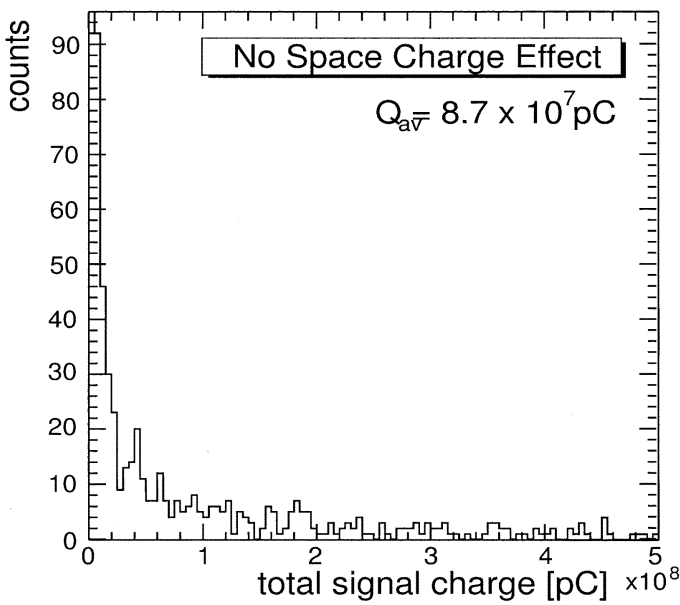

(a)

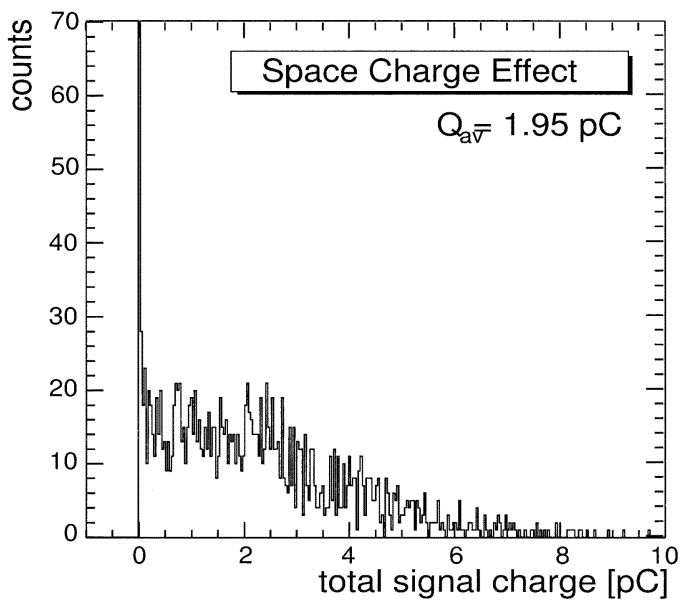

(b)

Fig. 4. Simulated charge spectra for a single gap timing RPC at $3 \mathrm{kV}$, $p=1013 \mathrm{mb}$ and $T=296.15 \mathrm{~K}$. The mean charge is indicated $\left(Q_{\mathrm{av}}\right)$. (a) Space charge effect is not included in the simulation. (b) Space charge effect is included.

serves a region where the charge is proportional to the primary charge (proportional mode). Here, the charge increases exponentially with the high voltage. After this one encounters the very narrow space charge mode of usually less than $100 \mathrm{~V}$, where that proportionality is no longer valid. When further increasing the high voltage, the average charge suddenly increases by a factor 10 to 100 (limited streamer mode). The charge continues to rise more slowly up to the general breakdown of the chamber or the Geiger-Müller mode, [20], [21].

For parallel plate geometries like RPCs and neglecting space charge effects, we expect an exponential dependence of the charge on $\alpha_{\text {eff }}(E)$. Since at high fields the dependence of $\alpha_{\mathrm{eff}}(E)$ on the field $E$ is approximately linear, the relation between the charge and $E$ will be approximately exponential, as in the wire chamber. As can be seen in Fig. 6(a), the timing RPC shows this proportional mode at low fields, which is, however, giving charges that are too small for efficient operation. We also observe that in the broad operational region from around 9 to $11 \mathrm{kV} / \mathrm{mm}$, the detector is operated in space charge mode.

In Fig. 6(b), we observe that the value of the charge depends first exponentially on the applied high voltage, but then the de-

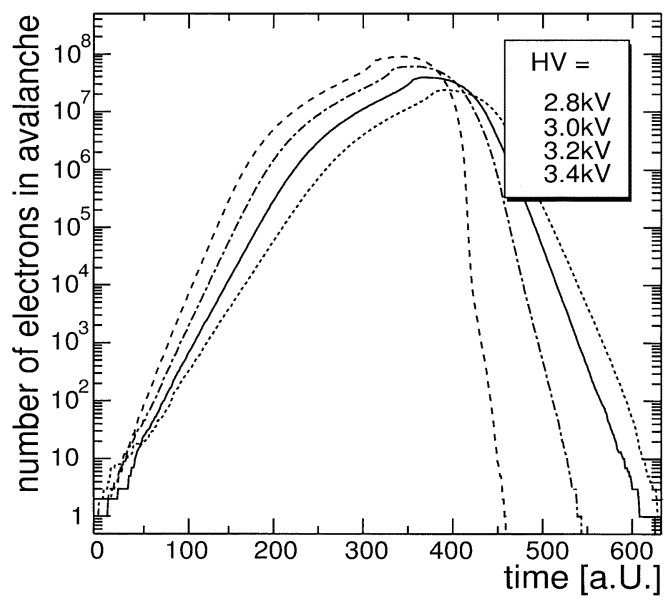

(a)

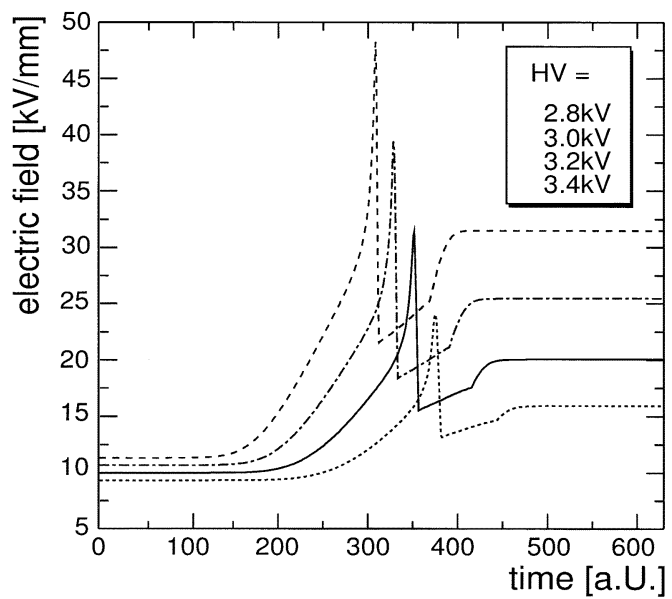

(b)

Fig. 5. (a) The total number of charge carriers in simulated avalanches started by one electron at the cathode for different high voltages. (b) The maximum value of the electric field in the same avalanches as in (a). 100 time steps correspond to about $0.5 \mathrm{~ns}$. The maximum is reached just before the electron cloud reaches the anode. From that point on the highest field is present at the tail of the electron cloud. After the electrons have left the gas gap their presence in the resistive anode layer and the ions in the gap still alter the electric field in the gap.

pendence becomes approximately linear, which is also an observed experimental fact (For example, see [22, Fig. 3]). Only at very high fields, the occurrence of streamers is experimentally observed [4], which limits the space charge region toward higher voltages.

The ratio of induced and total signal charge should be a constant if we neglect space charge effects [3], [7]

$$
\frac{Q_{\text {ind }}}{Q_{\text {tot }}}=\frac{\left|\vec{E}_{w}\right|}{\alpha} .
$$

Fig. 7 shows the value of $\alpha \cdot Q_{\text {ind }} / Q_{\text {tot }}$ at different field strengths. We see that it is a clear indicator for space charge effects. While in proportional mode it gives $\left|\vec{E}_{w}\right|=1.25 / \mathrm{mm}$ as expected, it strongly deviates from this value starting at a field of $7.5 \mathrm{kV} / \mathrm{mm}$. 


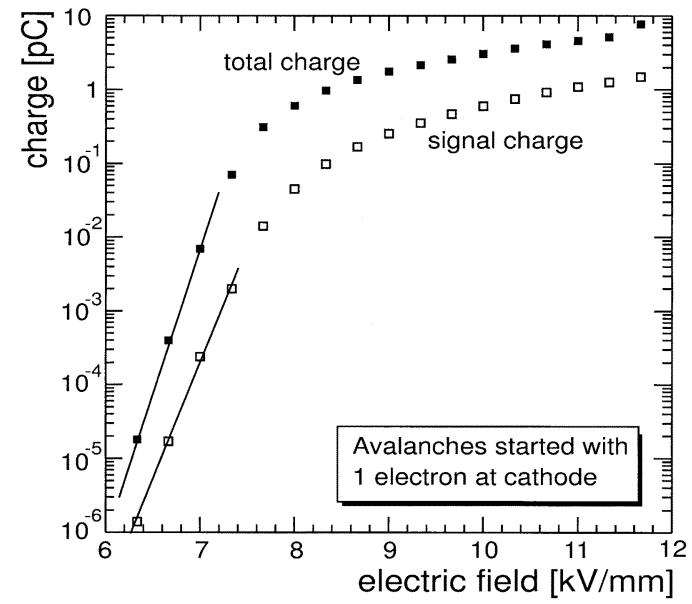

(a)

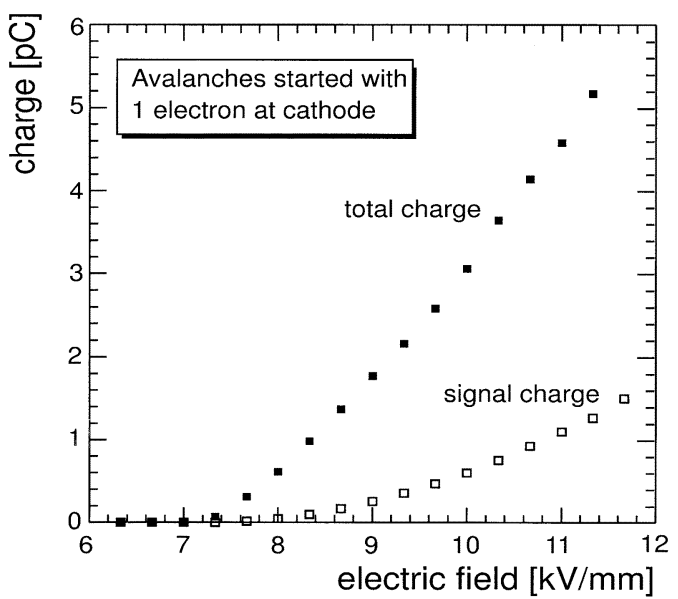

(b)

Fig. 6. (a) The total signal charge and the induced charge of simulated average avalanches started by one electron at the cathode as a function of the applied electric field. The proportional mode, where the charge is increasing exponentially with the high voltage, is indicated. It is, however, giving small charges. (b) The same plot on a linear scale. The approximately linear increase of the charges with the high voltage is visible.

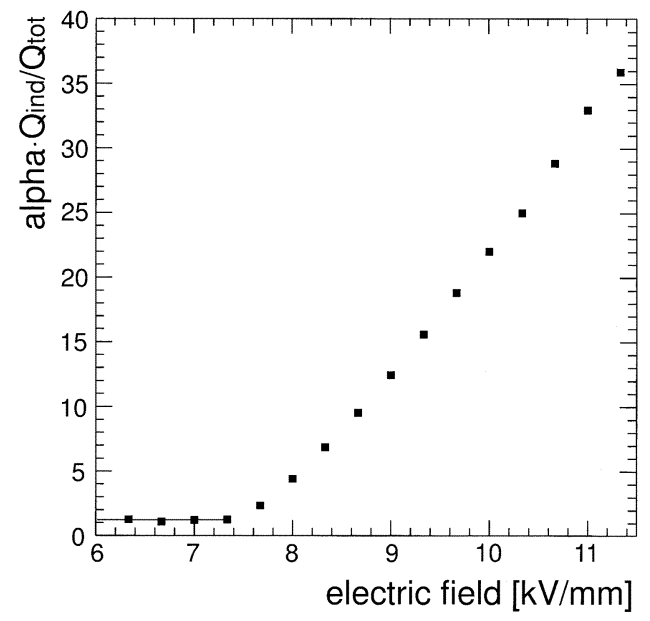

Fig. 7. The value of $\alpha \cdot Q_{\text {ind }} / Q_{\text {tot }}$ as a function of the applied electric field.

\section{CONCLUSION}

We have applied standard detector physics simulations to timing RPCs. A space charge effect is included by calculating dynamically the electric field of the avalanche charges and with this the values of the parameters important for the avalanche propagation: Townsend and attachment coefficients and drift velocity. Diffusion is also implemented. The simulated charge spectra of timing RPCs with a 0.3-mm single gap show a shape and average charges similar to measured spectra. A very strong space charge effect leads to a reduction of the observed charges by a factor $10^{7}$. Contrary to wire chambers, RPCs operate in space charge mode which is very broad. The experimental result of a first exponential and then linear dependence of the average charges on the high voltage is reproduced by the simulations. As expected, the simulations do not reproduce quantitatively the avalanche-to-streamer transition, because no photonic effects are included.

\section{ACKNOWLEDGMENT}

The authors would like to thank B. Schnizer, Technical University Graz, Graz, Austria, for his work on the electrostatic theory and P. Fonte, Laboratory for Instrumentation and Experimental Particles Physics, Coimbra, Portugal) for many interesting discussions.

\section{REFERENCES}

[1] E. C. Zeballos et al., "A new type of resistive plate chamber: The multigap RPC," Nucl. Instrum. Methods, vol. A 374, pp. 132-136, 1996.

[2] P. Fonte, R. F. Marques, J. Pinhão, N. Carolino, and A. Policarpo, "Highresolution RPC's for large TOF systems," Nucl. Instrum. Methods, vol. A 449, pp. 295-301, 2000.

[3] W. Riegler, R. Veenhof, and C. Lippmann, "Detector Physics and Simulation of Resistive Plate Chambers," CERN-EP-2002-046, to be published.

[4] P. Camarri, R. Cardarelli, A. Di Ciacco, and R. Santonico, "Streamer suppression with $\mathrm{SF}_{6}$ in RPC's operated in avalanche mode," Nucl. Instrum. Methods, vol. A 414, pp. 317-324, 1998.

[5] A. Akindinov et al., "A four-gap glass-RPC time-of-flight array with 90-ns time resolution," IEEE Trans. Nucl. Sci., vol. 48, pp. 1658-1663, Oct. 2001.

[6] P. Fonte and V. Peskov, "High Resolution TOF With RPC's," LIP/00-04, 2000.

[7] H. Räther, Electron Avalanches and Breakdown in Gases. Washington, DC: Butterworth, 1964

[8] C. Lippmann and W. Riegler. Space charge effects and induced signals in resistive plate chambers. presented at Proc. 6th Int. Conf. Resistive Plate Chambers and Related Detectors. [Online]. Available: http://www.coimbra.lip.pt/ rpc2001/papers.html

[9] M. Abbrescia, "The simulation of resistive plate chambers in avalanche mode: Charge spectra and efficiency," Nucl. Instrum. Methods, vol. A 431, pp. 413-427, 1999.

[10] P. Fonte, "High resolution timing of MIPS with RPCs-A model," Nucl. Instrum. Methods, vol. A 456, pp. 6-10, 2000.

[11] I. Smirnov, HEED, Program to Compute Energy Loss of Particle in Gases, Version 1.01. Geneva, Switzerland: CERN, 1999.

[12] S. Biagi, MAGBOLTZ, Program to Compute Gas Transport Parameters, Version 2.2. Geneva, Switzerland: CERN, 2000.

[13] — IMONTE, Program to Compute Gas Properties, Version 4.5. Geneva, Switzerland: CERN, 2002.

[14] T. Heubrandtner, B. Schnizer, C. Lippmann, and W. Riegler, "Static Electric Fields in an Infinite Plane Condensor With One or Three Homogeneous Layers,", Geneva, Switzerland, CERN-OPEN, no. 74, 2001.

[15] _ - "Static electric fields in an infinite plane condensor with one or three homogeneous layers," Nucl. Instrum. Methods, vol. A 489, pp. 439-443, 2002. 
[16] W. Riegler, "Induced signals in resistive plate chambers," Nucl. Instrum. Methods, vol. A 491, pp. 258-271, 2002.

[17] "Addendum to the Technical Design Report of the Time-of-Flight System (TOF)," The ALICE collaboration, Geneva, Switzerland, CERN-LHCC-2000-016, ALICE-TDR-8-ADD-1, 2002.

[18] P. Fonte, "A model for breakdown in parallel plate chambers," IEEE Trans. Nucl. Sci., vol. 43, pp. 2135-2140, June 1996.

[19] P. Fonte, V. Peskov, and B. D. Ramsey, "Streamers in MSGC's and other gaseous detectors," ICFA Instrumentation Bulletin, vol. 15, pp. 1-13, 1997.
[20] W. Blum and L. Rolandi, Particle Detection With Drift Chambers. Berlin, Germany: Springer-Verlag, 1993.

[21] M. Atac et al., "Self-quenching streamers," Nucl. Instrum. Methods, vol. 200, p. 345, 1982.

[22] G. Aielli et al., "RPC ageing studies," Nucl. Instrum. Methods, vol. A 478, pp. 271-276, 2002. 